\section{A Prospective Trial Determin- ing the Relationship between Corneal Hysteresis, Corneal Corrected Intraocular Pressure, and Optical Coherence Tomog- raphy Parameters in Open Angle Glaucoma Suspects}

\section{Ian McWherter ${ }^{1,2,3^{*}}$, Fraser McKay ${ }^{4}$, Paul Karpecki ${ }^{4}$, Meg Wright Sidle ${ }^{5,6,7}$ and Thomas A Jackson ${ }^{8}$}

${ }^{1}$ Director of Research, Kentucky School of Optometry, University of Pikeville, $K Y, U S A$

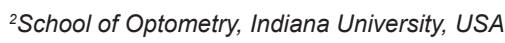

${ }^{3}$ Consultative Optometrist, Bennett and Bloom Eye Centers, Louisville, KY, USA

${ }^{4}$ Kentucky College of Optometry, University of Pikeville, KY, USA

${ }^{5}$ University of Pikeville, KY, USA

${ }^{6}$ Director of Institutional Research and Effectiveness, University of Pikeville, $K Y, U S A$

${ }^{7}$ Athletic Compliance Administrator, NAIA, USA

${ }^{8}$ Independent Researcher, USA

\begin{abstract}
Background: Glaucoma has been associated with low corneal hysteresis, a characteristic that may provide additional information in identifying those who are considered glaucoma suspects. The current study aimed to determine the relationship between corneal hysteresis, corneal compensated intraocular pressure, and GCC analysis in patients who are labeled clinically as open angle glaucoma suspects.
\end{abstract}

Methods: Participants were categorized into 2 groups, control $(n=14)$

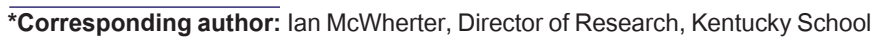
of Optometry, University of Pikeville, KY, USA; SSchool of Optometry, Indiana University, USA, Tel: +1 7242444644; Fax: +1 5023614488; E-mail: ianmcwherter@upike.edu

Citation: McWherter I, McKay F, Karpecki P, Sidle MW, Jackson TA (2018) A Prospective Trial Determining the Relationship between Corneal Hysteresis, Corneal Corrected Intraocular Pressure, and Optical Coherence Tomography Parameters in Open Angle Glaucoma Suspects. J Ophthalmic Clin Res 4: 044.

Received: May 30, 2018; Accepted: June19, 2018; Published: July 03, 2018

Copyright: (C) 2018 McWherter I, et al. This is an open-access article distributed under the terms of the Creative Commons Attribution License, which permits unrestricted use, distribution, and reproduction in any medium, provided the original author and source are credited.

\begin{abstract}
or suspect $(n=27)$, based on clinical examination. Corneal hysteresis and optical coherence tomography were performed and analyzed for any correlation between these two groups.

Results: Correlations between corneal hysteresis and parapapillary retinal nerve fiber layer thickness or corneal hysteresis and ganglion cell complex thickness in the macula were weak to moderate and ranged from $r=-0.340$ to $r=0.316$. The correlations between corneal compensated intraocular pressure and parapapillary retinal nerve fiber layer thickness or corneal compensated intraocular pressure and ganglion cell complex thickness were also weak to moderate and ranged from $r=-0.489$ to $r=0.396$.

Conclusion: In the current study, corneal hysteresis and corneal compensated intraocular pressure did not provide a definitive relationship when trying to identify patients as glaucoma suspects. The relationships between corneal hysteresis and retinal nerve fiber layer thickness or corneal hysteresis and ganglion cell complex thickness were weak to moderate. The weak to moderate correlation was consistent even when considering retinal nerve fiber layer and ganglion cell complex global indices as well as the individual clock hours for both measurements.
\end{abstract}

Keywords: Corneal compensated intraocular pressure; Corneal hysteresis; Open angle glaucoma suspect

\section{Introduction}

Corneal hysteresis is a corneal biomechanical property that is related to the viscoelasticity of the cornea. Viscoelasticity is a somewhat complicated physical principle that describes how something that absorbs energy maintains its characteristics by acting both as an elastic system and dampener, converting some energy to heat. The corneal hysteresis and its role in glaucoma and other ocular pathology, such as Keratoconus and Fuch's Dystrophy, has been explored in great detail of late thanks to the Ocular Response Analyzer (ORA, [Reichert Inc, Buffalo, NY]) [1-3].

The ocular response analyzer is a non-contact tonometer that measures corneal biomechanical properties and intraocular pressure. In addition to measuring corneal hysteresis and the Goldmann-correlated intraocular pressure, the ocular response analyzer also measures the corneal compensated intraocular pressure. Corneal compensated intraocular pressure is of special interest because unlike Goldman applanation tonometry; it has been found to be non-dependent on central corneal thickness $[4,5]$. The corneal compensated intraocular pressure correlates well with Goldman applanation tonometry in healthy eyes; however, glaucomatous eyes have been shown to have a higher corneal compensated intraocular pressure when compared to Goldman applanation tonometry [6]. This difference is most pronounced in normal tension glaucoma patients where the disparity between corneal compensated intraocular pressure and Goldman applanation tonometry can be up to $5 \mathrm{mmHg}$ [6].

Spectral Domain Optical Coherence Tomography (OCT, Cirrus HD-OCT [Carl Zeiss Meditec, Dublin, CA]) obtains 3-D data cubes of the optic disc and macula for quantitative assessment to include 
parapapillary Retinal Nerve Fiber Layer ( RNFL) thickness and the Ganglion Cell Complex thickness (GCC) [7]. The ganglion cells within the inner retina contain axons that comprise the RNFL. RNFL loss is a well-recognized sign of early glaucoma. Recently, imaging of the layer containing the ganglion cell bodies and their dendrites has become commercially available. GCC imaging is a promising technology since damage to the ganglion cells should theoretically precede the loss of the retinal nerve fiber layer [7].

A challenging aspect of patient care in glaucoma is arriving at the diagnosis of glaucoma from glaucoma suspect. While the diagnosis may be clear at times, it frequently is not. With suspicious characteristics or findings still present, patients are often labeled glaucoma suspects. Technology such as spectral domain optical coherence tomography has improved the diagnostic accuracy, but additional confirmatory testing would be helpful in making this difficult determination. The purpose of this investigation is to determine the relationship between corneal hysteresis, corneal compensated intraocular pressure, and GCC analysis in patients who are labeled clinically as open angle glaucoma suspects versus a control group.

\section{Methods}

Clinical definition of glaucoma suspect has been described elsewhere [8] and includes: Goldman applanation tonometry of greater than or equal to $22 \mathrm{mmHg}$, and/or cup to disk ratio of greater than or equal to 0.5 with the appearance of glaucomatous optic nerve damage (i.e., thinning or notching), and/or a cup to disc asymmetry of greater than or equal to 0.2 . For complete inclusion/exclusion criteria (Table 1).

Consecutive patients that presented for an eye examination were asked to participate in the study. If both eyes of a normal patient were eligible, both eyes were included in the study. Consecutive patients who presented for an eye exam and were determined to be a glaucoma suspect or have been determined to be a glaucoma suspect on a previ ous visit were asked to participate in the study. All patients signed an informed consent in accordance with the Declaration of Helsinki; the Schulman Institutional Review board approved the study.

Willing patients underwent a baseline examination. This included best corrected visual acuity, a detailed ocular, family, and medical history, blood pressure, pulse, pupil and extra-ocular muscle testing, Goldmann intraocular pressure (average of 2 measurements), slit lamp exam, gonioscopy, corneal pachymetry, Ocular Response Analyzer testing (average of 2 measurements), dilated fundus exam, spectral domain optical coherence tomography testing of both retinal nerve fiber layer and ganglion cell complex, and 24-2 Humphrey SITA standard visual field testing. The study used the reference database on the Cirrus 5.0 optical coherence tomography to define thinning of the retinal nerve fiber layer and the ganglion cell complex. Optical coherence tomography testing was repeated to confirm defects. Only signal strength of 8 or greater on optical coherence tomography testing and 6.5 or greater on Ocular Response Analyzer testing was accepted. Visual field tests included in the study had less than $33 \%$ false positive, false negative results, and fixation loss and the reference database on the Humphrey visual field was used to determine normal versus abnormal values. Any visual field depression was repeated 2-4 weeks later to confirm its reproducibility. If a visual field abnormality was confirmed the patient was excluded from the study and treatment was initiated appropriately.

The optic nerve head parameters were confirmed with optical coherence tomography testing to reduce intra- and inter- observer variability. The eye that met the criteria to be considered a glaucoma suspect was used in the study; if both eyes met the criteria, then both eyes were used in the study. However, if either eye in a patient is deemed to be a glaucoma suspect the other eye was not used as a normal control. Inclusion/Exclusion criteria are included in table 1. Specifically, patients with mixed mechanism glaucoma, previous ocular surgery, and coexisting ocular pathology were excluded from the study.

\begin{tabular}{|c|c|c|}
\hline Control & Glaucoma Suspect Inclusion & Exclusion \\
\hline Goldmann IOP between 11-21 mmHg & Goldmann IOP $>/=22 \mathrm{mmHg}$ & BCVA worse than $6 / 12$ in either eye \\
\hline $\mathrm{CD}</=0.4$ & $\mathrm{CD}$ ratio $>/=0.5$ with glaucomatous nerve damage & Hx of angle closure/recession or occludable angle on gonioscopy \\
\hline \multirow[t]{9}{*}{$\begin{array}{l}\text { No first degree } \\
\text { FxHx of Glaucoma }\end{array}$} & Open angle on Gonioscopy & Previous SLT/ALT/LPI in either eye \\
\hline & Age [25-82] & Any previous treatment with topical/oral glaucoma medication \\
\hline & Ability to speak, read and understand English & Currently active uveitis, Hx of uveitis \\
\hline & & Corneal dystrophy, ulcer or infection \\
\hline & & Hx of overnight contact lens wear \\
\hline & & Visual field loss (regardless of etiology) \\
\hline & & Macular/Retinal pathology \\
\hline & & $\begin{array}{l}\text { Systemic medication with IOP effects (corticosteroids, carbonic } \\
\text { anhydrase inhibitors, etc) }\end{array}$ \\
\hline & & $\begin{array}{l}\text { Women who are or intend to become pregnant in next } 4 \text { months } \\
\text { (patient reported) }\end{array}$ \\
\hline
\end{tabular}

Table 1: Inclusion/Exclusion.

IOP- Intraocular Pressure; CD- Cup-to-Disc; FxHx- Family History; BCVA- Best Corrected Visual Acuity; PXF- Pseudo Exfoliation Syndrome; PDS- Pigment Dispersion Syndrome; SLT- Selective Laser Trabeculoplasty; ALT- Argon Laser Trabeculoplasty; LPI- Laser Peripheral Iridotomy; Macular Pathology defined as but not limited to Diabetic retinopathy, Age related macular degeneration, Vitreomacular traction, Macular hole, Epiretinal membrane 


\section{Statistical Analysis}

All collected data was analyzed computing a combination of descriptive statistics and correlation coefficients using SPSS v24. The achieved power was 0.93 (1- $\beta$ error probability) given $\alpha$ of 0.05 and a sample size of 70 . For all analyses, the nasal and temporal quadrants were correlated between the right and left eye and all clock hours are referenced to the right eye in order to aid in interpretation. The statistical methods used in this study included frequency distributions, means, standards deviations, and Pearson correlation coefficients.

\section{Results}

The study enrolled a total of 70 eyes (27 control and 43 glaucoma suspect). Gender distribution was similar between the two groups, however, the age and racial distributions of the two groups were uneven (Table 2). The average age of patients in the control group was 37 years old (SD 14.39) and the average age in the glaucoma suspect group was 59 years old (SD 13.31). While the control group was overwhelmingly Caucasian (71 percent), the second group was mixed between Caucasian and African American with some other races as well.

\begin{tabular}{|c|c|c|}
\hline & Control & $\begin{array}{c}\text { Glaucoma } \\
\text { Suspect }\end{array}$ \\
\hline Number of Unduplicated Participants & 14 & 27 \\
\hline Average Age (SD) & $37.00(14.39)$ & $59.37(13.31)$ \\
\hline Gender Male & $35.7 \%$ & $33.3 \%$ \\
\hline Female & $64.3 \%$ & $66.7 \%$ \\
\hline Race Aaucasian & $71.4 \%$ & $59.3 \%$ \\
\hline African American & 21.4 & 33.3 \\
\hline Asian & 7.2 & 3.7 \\
\hline Hicanic & 0.0 & 3.7 \\
\hline
\end{tabular}

Table 2: Unduplicated participant demographics.

Based on the data obtained, the relationships were weak to moderate between corneal hysteresis and RNFL or corneal hysteresis and GCC in the suspect and control groups, with correlations ranging from $r=-0.340$ to $r=0.316$ (Table 3 ). The weak to moderate correlation were found when considering RNFL and GCC global indices as well as the individual clock hours for both RNFL and GCC.

The relationships were mixed between corneal compensated intraocular pressure and RNFL or corneal compensated intraocular pressure and GCC in the suspect and control groups, with correlations ranging from $r=-0.489$ to $r=0.396$ (Table 4). The corneal compensated intraocular pressure correlation to individual RNFL clock hours found trends that were of interest. Specifically, RNFL clock hour 12 provided a weak negative correlation for the suspect group $(r$ $=-0.259)$ compared to a stronger positive moderate correlation in the control group $(r=0.329)$. Similarly, RNFL clock hour 1 o'clock provided a weak negative correlation for the suspect group $(r=-0.245)$ compared to a stronger positive moderate correlation in the control group $(r=0.396, \rho=0.041)$. Something that surprised the authors was that while the suspect group had a weak negative correlation at RNFL clock hour 9 o'clock $(r=-0.183)$, the control group had a moderately strong negative correlation $(r=-0.489, \rho=0.010)$ at the same position.
Similar analysis was performed with Goldmann applanation tonometry vs RNFL thickness, with correlations ranging from $r=-0.393$ to $r=0.316$ (Table 5). The overall thickness showed a moderate negative correlation in the suspect group $(r=-0.343, \rho=0.025)$, with a stronger negative correlations at RNFL clock hour 5 o'clock and 6 o'clock ( $r=-0.390, \rho=0.008$; and $r=-0.393, \rho=0.008$ respectively). However, the rest of the hourly comparisons of the RNFL thickness vs. Goldmann applanation tonometry were not consistent and did not match the corneal compensated intraocular pressure correlation.

\section{Discussion}

Identifying glaucomatous risk factors early in the life of a patient is important, not only for those who require treatment and regular follow ups, but to help lessen repetitive testing in those that may not share the same anatomical characteristics as others. Corneal hysteresis has provided another metric that can quantify the biomechanical properties within a cornea and its relationship with other anatomical/ physiological variations. The role of optical coherence tomography and early glaucoma detection are well established; both retinal nerve fiber layer thickness and ganglion cell complex play a vital role in monitoring irreversible damage caused by glaucoma. The purpose of this investigation was to determine the relationship between corneal hysteresis/corneal compensated intraocular pressure and current optical coherence tomography technology in patients labeled clinically as open angle glaucoma suspects.

Given the relationship between corneal hysteresis and corneal compensated intraocular pressure to glaucoma $[9,10]$ the authors were surprised to find that corneal hysteresis did not appear to have any strong relationship with glaucoma suspects in regards to the RNFL and GCC measurements when compared to the control group. While corneal compensated intraocular pressure correlations in certain RNFL clock hours reached statistical significance, these were weak to moderate correlations and were not consistent throughout the analysis. These inconsistent results may be explained by limitations of current corneal hysteresis and OCT technologies, such measurement variability, segmentation algorithms, and/or potential study bias from utilizing both eyes of study patients. Due to the weak to only moderate correlations found in the current study, it appeared that corneal hysteresis and corneal compensated intraocular pressure did not provide a definitive relationship when trying to identify patients as glaucoma suspects. There may be merit in evaluating the same group over time, or patients already diagnosed with glaucoma, and examining biomechanics trends in those who progress compared to those who do not.

Limitations of the current study include its small sample size, uneven patient demographics, and limited progression analysis. The authors suggest that the results of the current study be confirmed with a larger multicenter prospective trial that includes a more diverse patient population. Despite the best efforts to quantify glaucoma suspects, certain criteria are inevitably subjective and cloud any statistical analysis. This highlights both the difficulty in studying and need for more definitive measures in labeling those as glaucoma suspects.

\section{Acknowledgement}

The authors would like to thank Reichert, inc. for providing funding and resources to make this study possible. 
Citation: McWherter I, McKay F, Karpecki P, Sidle MW, Jackson TA (2018) A Prospective Trial Determining the Relationship between Corneal Hysteresis, Corneal Corrected Intraocular Pressure, and Optical Coherence Tomography Parameters in Open Angle Glaucoma Suspects. J Ophthalmic Clin Res 4: 044.

- Page 4 of 5 •

\begin{tabular}{|c|c|c|c|c|}
\hline & Mean (SD) & $\begin{array}{c}\text { Correlation with Baseline Corneal } \\
\text { Hysteresis - SUSPECT GROUP (Sig.) }\end{array}$ & Mean (SD) & $\begin{array}{l}\text { Correlation with Baseline Corneal Hyster- } \\
\text { esis - CONTROL GROUP (Sig.) }\end{array}$ \\
\hline Number of Eyes & & 43 & & 27 \\
\hline Corneal Hysteresis & $12.58(11.74)$ & & $11.54(1.57)$ & $r=-0.040(\rho=0.841)$ \\
\hline Baseline OCT average RNFL & $93.76(20.00)$ & $r=0.032(\rho=0.837)$ & $93.50(10.99)$ & $r=-0.118(\rho=0.556)$ \\
\hline Baseline 12 o'clock RNFL & $113.69(30.00)$ & $r=0.148(\rho=0.331)$ & $109.00(29.85)$ & $r=-0.177(\rho=0.377)$ \\
\hline Baseline 1 o'clock RNFL & $99.51(22.39)$ & $r=0.065(\rho=0.670)$ & $101.19(24.66)$ & $r=0.251(\rho=0.207)$ \\
\hline Baseline 2 o'clock RNFL & $88.60(17.92)$ & $r=0.106(\rho=0.487)$ & $86.70(18.66)$ & $r=0.172(\rho=0.390)$ \\
\hline Baseline 3 o'clock RNFL & $62.49(10.20)$ & $r=-0.090(\rho=0.557)$ & $56.15(9.97)$ & $r=0.064(\rho=0.749)$ \\
\hline Baseline 4 o'clock RNFL & $66.69(14.22)$ & $r=0.055(\rho=0.718)$ & $65.44(9.52)$ & $r=0.109(\rho=0.588)$ \\
\hline Baseline 5 o'clock RNFL & $91.47(20.67)$ & $r=0.023(\rho=0.883)$ & $101.37(23.85)$ & $r=0.028(\rho=0.889)$ \\
\hline Baseline 6 o'clock RNFL & $121.64(31.68)$ & $r=-0.038(\rho=0.802)$ & $136.48(36.82)$ & $r=-0.340(\rho=0.083)$ \\
\hline Baseline 7 o'clock RNFL & $127.78(24.51)$ & $r=0.115(\rho=0.452)$ & $133.81(23.87)$ & $r=-0.083(\rho=0.682)$ \\
\hline Baseline 8 o'clock RNFL & $67.13(20.14)$ & $r=0.181(\rho=0.233)$ & $68.74(14.37)$ & $r=0.069(\rho=0.733)$ \\
\hline Baseline 9 o'clock RNFL & $51.07(10.45)$ & $r=-0.238(\rho=0.115)$ & $54.93(17.23)$ & $r=-0.053(\rho=0.793)$ \\
\hline Baseline 10 o'clock RNFL & $78.11(19.01)$ & $r=0.014(\rho=0.928)$ & $83.07(24.56)$ & $r=0.047(\rho=0.815)$ \\
\hline Baseline 11 o'clock RNFL & $124.60(23.83)$ & $r=0.068(\rho=0.656)$ & $125.56(25.32)$ & $r=0.260(\rho=0.209)$ \\
\hline Baseline GCC average & $78.47(8.89)$ & $r=0.231(\rho=0.128)$ & $81.20(12.32)$ & $r=0.164(\rho=0.433)$ \\
\hline Baseline GCC minimum & $75.73(10.00)$ & $r=0.155(\rho=0.308)$ & $80.08(13.35)$ & $r=0.272(\rho=0.188)$ \\
\hline Baseline Sup GCC & $79.00(9.03)$ & $r=0.244(\rho=0.107)$ & $81.20(13.17)$ & $r=0.316(\rho=0.124)$ \\
\hline Baseline Sup temp GCC & $79.20(8.22)$ & $r=0.168(\rho=0.271)$ & $80.32(11.58)$ & $r=0.216(\rho=0.300)$ \\
\hline Baseline Inf temp GCC & $78.73(8.74)$ & $r=0.161(\rho=0.290)$ & $80.92(10.98)$ & $r=0.167(\rho=0.426)$ \\
\hline Baseline Inf GCC & $74.69(13.83)$ & $r=0.138(\rho=0.367)$ & $78.96(12.30)$ & $r=0.281(\rho=0.174)$ \\
\hline Baseline Inf nasal GCC & $78.33(9.81)$ & $r=0.215(\rho=0.156)$ & $82.12(12.78)$ & $r=0.253(\rho=0.222)$ \\
\hline Baseline Sup nasal GCC & $79.49(10.13)$ & $r=0.257(\rho=0.089)$ & $82.52(12.45)$ & \\
\hline
\end{tabular}

Table 3: Correlations - Corneal hysteresis and RNFL, Corneal hysteresis and GCC.

\begin{tabular}{|c|c|c|c|c|}
\hline & Mean (SD) & $\begin{array}{l}\text { Correlation with Baseline corneal } \\
\text { compensated intraocular pressure - } \\
\text { SUSPECT GROUP (Sig.) }\end{array}$ & Mean (SD) & $\begin{array}{l}\text { Correlation with Baseline corneal } \\
\text { compensated intraocular pressure } \\
\text { CONTROL GROUP (Sig.) }\end{array}$ \\
\hline Number of Eyes & & 43 & & 27 \\
\hline Corneal Compensated Intraocular Pressure & $18.68(4.72)$ & & $15.08(3.55)$ & \\
\hline Baseline OCT average RNFL & $93.76(20.00)$ & $r=-0.113(\rho=0.471)$ & $93.50(10.99)$ & $r=-0.201(\rho=0.314)$ \\
\hline Baseline 12 o'clock RNFL & $113.69(30.00)$ & $r=-0.259(\rho=0.085)$ & $109.00(29.85)$ & $r=0.329(\rho=0.094)$ \\
\hline Baseline 1 o'clock RNFL & $99.51(22.39)$ & $r=-0.245(\rho=0.104)$ & $101.19(24.66)$ & $r=0.396(\rho=0.041)$ \\
\hline Baseline 2 o'clock RNFL & $88.60(17.92)$ & $r=-0.076(\rho=0.620)$ & $86.70(18.66)$ & $r=0.160(\rho=0.424)$ \\
\hline Baseline 3 o'clock RNFL & $62.49(10.20)$ & $r=-0.015(\rho=0.921)$ & $56.15(9.97)$ & $r=-0.270(\rho=0.174)$ \\
\hline Baseline 4 o'clock RNFL & $66.69(14.22)$ & $r=-0.205(\rho=0.178)$ & $65.44(9.52)$ & $r=-0.036(\rho=0.857)$ \\
\hline Baseline 5 o'clock RNFL & $91.47(20.67)$ & $r=-0.238(\rho=0.115)$ & $101.37(23.85)$ & $r=-0.099(\rho=0.625)$ \\
\hline Baseline 6 o'clock RNFL & $121.64(31.68)$ & $r=-0.256(\rho=0.089)$ & $136.48(36.82)$ & $r=-0.282(\rho=0.154)$ \\
\hline Baseline 7 o'clock RNFL & $127.78(24.51)$ & $r=-0.216(\rho=0.154)$ & $133.81(23.87)$ & $r=-0.370(\rho=0.058)$ \\
\hline Baseline 8 o'clock RNFL & $67.13(20.14)$ & $r=0.043(\rho=0.779)$ & $68.74(14.37)$ & $r=-0.368(\rho=0.059)$ \\
\hline Baseline 9 o'clock RNFL & $51.07(10.45)$ & $r=-0.183(\rho=0.229)$ & $54.93(17.23)$ & $r=-0.489(\rho=0.010)$ \\
\hline Baseline 10 o'clock RNFL & $78.11(19.01)$ & $r=-0.163(\rho=0.285)$ & $83.07(24.56)$ & $r=-0.291(\rho=0.141)$ \\
\hline Baseline 11 o'clock RNFL & $124.60(23.83)$ & $r=-0.229(\rho=0.130)$ & $125.56(25.32)$ & $r=-0.127(\rho=0.527)$ \\
\hline Baseline GCC average & $78.47(8.89)$ & $r=-0.127(\rho=0.405)$ & $81.20(12.32)$ & $r=-0.242(\rho=.243)$ \\
\hline Baseline GCC minimum & $75.73(10.00)$ & $r=-0.137(\rho=0.371)$ & $80.08(13.35)$ & $r=-0.159(\rho=0.448)$ \\
\hline Baseline Sup GCC & $79.00(9.03)$ & $r=-0.133(\rho=0.384)$ & $81.20(13.17)$ & $r=-0.267(\rho=0.197)$ \\
\hline Baseline Sup temp GCC & $79.20(8.22)$ & $r=-0.206(\rho=0.174)$ & $80.32(11.58)$ & $r=-0.330(\rho=0.108)$ \\
\hline Baseline Inf temp GCC & $78.73(8.74)$ & $r=-0.158(\rho=0.301)$ & $80.92(10.98)$ & $r=-0.254(\rho=0.220)$ \\
\hline Baseline Inf GCC & $74.69(13.83)$ & $r=-0.037(\rho=0.808)$ & $78.96(12.30)$ & $r=-0.182(\mathrm{p}=0.384)$ \\
\hline Baseline Inf nasal GCC & $78.33(9.81)$ & $r=-0.139(\rho=0.363)$ & $82.12(12.78)$ & $r=-0.214(\rho=0.304)$ \\
\hline Baseline Sup nasal GCC & $79.49(10.13)$ & $r=-0.084(\rho=0.585)$ & $82.52(12.45)$ & $r=-0.233(\rho=0.262)$ \\
\hline
\end{tabular}

Table 4: Correlations - Corneal compensated intraocular pressure and RNFL, corneal compensated intraocular pressure and GCC. 
Citation: McWherter I, McKay F, Karpecki P, Sidle MW, Jackson TA (2018) A Prospective Trial Determining the Relationship between Corneal Hysteresis, Corneal Corrected Intraocular Pressure, and Optical Coherence Tomography Parameters in Open Angle Glaucoma Suspects. J Ophthalmic Clin Res 4: 044.

- Page 5 of 5 •

\begin{tabular}{|c|c|c|c|c|}
\hline & Mean (SD) & $\begin{array}{l}\text { Correlation with Baseline Goldmann Intra- } \\
\text { ocular Pressure-SUSPECT GROUP (Sig.) }\end{array}$ & Mean (SD) & $\begin{array}{l}\text { Correlation with Baseline Goldmann Intra- } \\
\text { ocular Pressure-CONTROL GROUP (Sig.) }\end{array}$ \\
\hline Number of Eyes & & 43 & & 27 \\
\hline Goldmann intraocular pressure & $18.39(5.16)$ & & $15.63(4.27)$ & \\
\hline Baseline OCT average RNFL & $93.76(20.00)$ & $r=-0.343(\rho=0.025)$ & $93.50(10.99)$ & $r=-0.274(\rho=0.166)$ \\
\hline Baseline 12 o'clock RNFL & $113.69(30.00)$ & $r=-0.174(\rho=0.252)$ & $109.00(29.85)$ & $r=0.045(\rho=0.822)$ \\
\hline Baseline 1 o'clock RNFL & $99.51(22.39)$ & $r=-0.141(\rho=0.355)$ & $101.19(24.66)$ & $r=0.164(\rho=0.414)$ \\
\hline Baseline 2 o'clock RNFL & $88.60(17.92)$ & $r=-0.246(\rho=0.104)$ & $86.70(18.66)$ & $r=0.151(\rho=0.451)$ \\
\hline Baseline 3 o'clock RNFL & $62.49(10.20)$ & $r=-0.209(\rho=0.169)$ & $56.15(9.97)$ & $r=-0.252(\rho=0.205)$ \\
\hline Baseline 4 o'clock RNFL & $66.69(14.22)$ & $r=-0.275(\rho=0.067)$ & $65.44(9.52)$ & $r=-0.249(\rho=0.210)$ \\
\hline Baseline 5 o'clock RNFL & $91.47(20.67)$ & $r=-0.390(\rho=0.008)$ & $101.37(23.85)$ & $r=-0.290(\rho=0.142)$ \\
\hline Baseline 6 o'clock RNFL & $121.64(31.68)$ & $r=-0.393(\rho=0.008)$ & $136.48(36.82)$ & $r=-0.302(\rho=0.126)$ \\
\hline Baseline 7 o'clock RNFL & $127.78(24.51)$ & $r=-0.184(\rho=0.225)$ & $133.81(23.87)$ & $r=-0.328(\rho=.095)$ \\
\hline Baseline 8 o'clock RNFL & $67.13(20.14)$ & $r=0.316(\rho=0.035)$ & $68.74(14.37)$ & $r=-0.198(\rho=0.322)$ \\
\hline Baseline 9 o'clock RNFL & $51.07(10.45)$ & $r=0.129(\mathrm{p}=0.400)$ & $54.93(17.23)$ & $r=-0.338(\rho=0.084)$ \\
\hline Baseline 10 o'clock RNFL & $78.11(19.01)$ & $r=-0.016(\rho=0.918)$ & $83.07(24.56)$ & $r=-0.179(\rho=0.373)$ \\
\hline Baseline 11 o'clock RNFL & $124.60(23.83)$ & $r=-0.099(\rho=.519)$ & $125.56(25.32)$ & $r=0.006(\mathrm{p}=0.976)$ \\
\hline Baseline GCC average & $78.47(8.89)$ & $r=-0.145(\rho=.342)$ & $81.20(12.32)$ & $r=-0.130(\rho=0.536)$ \\
\hline Baseline GCC minimum & $75.73(10.00)$ & $r=-0.103(\rho=0.502)$ & $80.08(13.35)$ & $r=-0.094(\rho=0.656)$ \\
\hline Baseline Sup GCC & $79.00(9.03)$ & $r=-0.123(\rho=0.422)$ & $81.20(13.17)$ & $r=-0.126(\rho=0.549)$ \\
\hline Baseline Sup temp GCC & $79.20(8.22)$ & $r=-0.170(\rho=.265)$ & $80.32(11.58)$ & $r=-0.191(\rho=0.361)$ \\
\hline Baseline Inf temp GCC & $78.73(8.74)$ & $r=-0.140(\rho=.358)$ & $80.92(10.98)$ & $r=-0.149(\rho=0.478)$ \\
\hline Baseline Inf GCC & $74.69(13.83)$ & $r=-0.151(\rho=.321)$ & $78.96(12.30)$ & $r=-0.136(\rho=0.516)$ \\
\hline Baseline Inf nasal GCC & $78.33(9.81)$ & $r=-0.148(\rho=0.330)$ & $82.12(12.78)$ & $r=-0.123(\rho=.558)$ \\
\hline Baseline Sup nasal GCC & $79.49(10.13)$ & $r=-0.085(\rho=0.578)$ & $82.52(12.45)$ & $r=-0.138(\rho=0.509)$ \\
\hline
\end{tabular}

Table 5: Correlations - Goldmann intraocular pressure and RNFL, Goldmann intraocular pressure and GCA.

\section{Conflicts of interest}

Dr. Paul Karpecki's financial disclosures include consultation work for Aerie Pharmaceuticals, Akorn, Alcon Labs, Allergan, Bausch + Lomb/Valeant, Glaukos, iCare USA, Katena, Konan Medical, Ocular Therapeutix, Oculus, Optometric Medical Solutions (OMS), Paragon Bioteck, Reichert, Shire Pharmaceuticals, Science Based Health, Sentiss Pharmaceuticals, and Topcon.

\section{References}

1. Cohen E (2009) Keratoconus and normal-tension glaucoma: A study of the possible association with abnormal biomechanical properties as measured by corneal hysteresis (An AOS Thesis). Trans Am Ophthalmol Soc 107: 282-299.

2. Shah S, Laiquzzaman M, Cunliffe I, Mantry S (2006) The use of the Reichert ocular response analyser to establish the relationship between ocular hysteresis, corneal resistance factor and central corneal thickness in normal eyes. Cont Lens Anterior Eye 29: 257-262.

3. Saad A, Lteif Y, Azan E, Gatinel D (2010) Biomechanical properties of Keratoconus Suspect Eyes. Invest Ophthalmol Vis Sci 51: 2912-2916.

4. De Moraes CV, Hill V, Tello C, Liebmann JM, Ritch R (2012) Lower corneal hysteresis is associated with more rapid glaucomatous visual field progression. J Glaucoma 21: 209-213.
5. Anand A, De Moraes CG, Teng CC, Tello C, Liebmann JM, et al. (2015) Corneal hysteresis and visual field asymmetry in open angle glaucoma. Invest Ophthalmol Vis Sci 51: 6514-6518.

6. Ehrlich JR, Radcliffe NM, Shimmyo M (2012) Goldmann applanation tonometry compared with corneal-compensated intraocular pressure in the evaluation of primary open-angle Glaucoma. BMC Ophthalmol 12: 52.

7. Hood DC, Raza AS, de Moraes CG, Liebmann JM, Ritch R (2013) Glaucomatous damage of the macula. Prog Retin Eye Res 32: 1-21.

8. Prum BE Jr, Lim MC, Mansberger SL, Stein JD, Moroi SE, et al. (2016) Primary Angle Glaucoma Suspect Preferred Practice Pattern ${ }^{(\circledR)}$ Guidelines. Ophthalmology 123: 112-151.

9. Prata TS, Lima VC, Guedes LM, Biteli LG, Teixeira SH, et al (2012) Association between corneal biomechanical properties and optic nerve head morphology in newly diagnosed glaucoma patients. Clin Experiment Ophthalmol 40: 682-688.

10. Wells AP, Garway-Heath DF, Poostchi A, Wong T, Chan KC, et al. (2008) Corneal hysteresis but not corneal thickness correlates with optic nerve surface compliance in glaucoma patients. Invest Ophthalmol Vis Sci 49: 3262-3268. 